\title{
Multi-Scale Observation of Hydrogen-Induced, Localized Plastic Deformation in Fatigue-Crack Propagation in a Pure Iron
}

\author{
Yuhei Ogawa ${ }^{1,2}$, Domas Birenis ${ }^{3}$, Hisao Matsunaga ${ }^{4,5,6}$, Annett Thøgersen $^{7}, \varnothing_{\text {ystein Prytz }}{ }^{3}$, \\ Osamu Takakuwa ${ }^{5}$ Junichiro Yamabe $e^{5,6,8,9}$ \\ ${ }^{1}$ Graduate School of Engineering, Kyushu University, \\ 744 Motooka, Nishi-ku, Fukuoka 819-0395, Japan \\ ${ }^{2}$ Research Fellow of the Japan Society for the Promotion of Science, \\ 744 Motooka, Nishi-ku, Fukuoka 819-0395, Japan \\ ${ }^{3}$ Department of Physics, Centre for Materials Science and Nanotechnology, University of Oslo, \\ PO Box 1048 - Blindern, NO-0316 Oslo, Norway \\ ${ }^{4}$ Department of Mechanical Engineering, Kyushu University, \\ 744 Motooka, Nishi-ku, Fukuoka 819-0395, Japan \\ ${ }^{5}$ Research Center for Hydrogen Industrial Use and Storage (HYDROGENIUS), Kyushu University, \\ 744 Motooka, Nishi-ku, Fukuoka 819-0395, Japan \\ ${ }^{6}$ International Institute for Carbon-Neutral Energy Research (I2CNER), Kyushu University, \\ 744 Motooka, Nishi-ku, Fukuoka 819-0395, Japan \\ ${ }^{7}$ SINTEF Materials and Chemistry, PO Box 124 - Blindern, NO-0314 Oslo, Norway \\ ${ }^{8}$ International Research Center for Hydrogen Energy, Kyushu University, \\ 744 Motooka, Nishi-ku, Fukuoka 819-0395, Japan \\ ${ }^{9}$ AIST-Kyushu University Hydrogen Materials Laboratory (HydroMate) \\ 744 Motooka, Nishi-ku, Fukuoka 819-0395, Japan
}

Corresponding Author: Yuhei Ogawa, E-mail: yuhei.ogawa.bz@gmail.com

\begin{abstract}
In order to study the influence of hydrogen on plastic deformation behavior in the vicinity of the fatigue crack-tip in a pure iron, a multi-scale observation technique was employed, comprising electron channeling contrast imaging (ECCI), electron back-scattered diffraction (EBSD) and transmission electron microscopy (TEM). The analyses successfully demonstrated that hydrogen greatly suppresses the dislocation structure evolution around the fracture path and localizes the plastic flow in the crack-tip
\end{abstract}


region. Such clear evidence can reinforce the existing model in which this type of localized plasticity contributes to crack-growth acceleration in metals in hydrogen atmosphere, which has not yet been experimentally elucidated.

\section{Keywords}

Fatigue, Hydrogen embrittlement, Dislocations, Scanning electron microscopy (SEM), Transmission electron microscopy (TEM).

Hydrogen-assisted fatigue crack-growth (HAFCG) in structural steels [1-4] is of significant concern, particularly with respect to the storage of compressed gaseous hydrogen in a futurehydrogen energy-based economy. In the presence of hydrogen, two types of cracking behavior are generally accepted in steels, i.e., stress-controlled fracture in high-strength steels and strain-controlled, quasi-static fracture in low- to medium-strength steels [5]. In the former case, hydrogen triggers brittle cracking along the grain boundaries and the intensity of crack acceleration becomes more pronounced with a lower loading rate (delayed fracture) [6,7]. In contrast, crack extension is mediated by a quantity of plastic flow and the fracture is frequently characterized by quasi-cleavage, showing no delayed cracking, as in the latter case [8]. Whereas several successful models have been proposed to demonstrate the behavior of stress-controlled fracture $[6,7]$, due to its complex nature, the detailed mechanisms of strain-controlled cracking are still not fully understood.

Extensive investigations for the strain-controlled HAFCG have also been conducted by the authors' research group, with the resulting proposal of one possible model to describe the series of phenomena; hydrogen-induced successive crack-growth (HISCG) [9-13]. The main role of hydrogen in this model is to concentrate plastic flow in crack-tip region, in accordance with the hydrogen-enhanced localized plasticity (HELP) [14, 15], leading to suppressed crack-tip blunting and faster crack propagation. However, critical evidence is still lacking to justify the use of the model, although similar mechanisms have also been implied by several researchers, based on the fractographic features of fatigue-tested or statically-cracked samples in hydrogen atmosphere [16, 17].

One of the reasons for this lack of strong evidence is that most of the experiments have been performed on materials with complex microstructures, several alloying elements and sometimes even two or more phases. All of these factors make interpretation difficult and thus, the analysis of the hydrogen effect on the fracture processes is further complicated. Hence, in this study, pure Fe was targeted in order to avoid those complications. Additionally, most of the past studies have been conducted either on macroor nano-scale, lacking any link between the phenomena of the two different scales. Dislocation structures have recently attracted attention as the key to understand the mechanism of hydrogen-induced degradation [18-23]. Transmission electron microscopy (TEM) is used for analyzing dislocation structures, but characteristics of the TEM samples (e.g., local plastic deformation and crack morphology) 
are often not clarified in as much detail as can be obtained via other techniques. In this study, we present an example of the successful utilization of electron channeling contrast imaging (ECCI) and orientation imaging microscopy (OIM), in combination with TEM imaging, with the aim of elucidating the crack-tip deformation in HAFCG on different scales. Specifically, ECCI and OIM are used to characterize crack morphology on a microscale, while TEM reveals the dislocation structures at specific locations on a smaller scale.

In order to demonstrate the hydrogen-enhanced, localized, plastic deformation in the fatigue crack-tip region, fatigue crack-growth (FCG) tests were performed in a hydrogen atmosphere. Various observation techniques were employed to prove that hydrogen dramatically reduces the plasticity expansion around the fatigue crack-tip, while significantly affecting dislocation structure evolution just beneath the fracture surface.

The material used was a commercial, pure Fe plate, with the chemical composition of $0.001 \% \mathrm{C}-0.07 \% \mathrm{Mn}-0.010 \% \mathrm{P}-0.003 \% \mathrm{~S}$. The yield stress, $\sigma_{\mathrm{Y}}$, and tensile strength, $\sigma_{\mathrm{B}}$ were $133 \mathrm{MPa}$ and $252 \mathrm{MPa}$, respectively. Compact-tension (CT) specimens with a width, $W$, of $50.8 \mathrm{~mm}$, and a thickness, $B$, of $10.0 \mathrm{~mm}$, were cut from L-T orientation of the plate.

FCG tests were performed in air and 0.7 MPa hydrogen at room temperature (RT), according to the ASTM E647 [24] standard, under a constant load range condition ( $\Delta P$-constant test), in order to identify the relationship between the FCG rate, $\mathrm{d} a / \mathrm{d} N$, and the stress intensity factor range, $\Delta K$. The load range, $R$, and the test frequency, $f$, were 0.1 and $1 \mathrm{~Hz}$, respectively. By using a low carbon steel, it was confirmed in our previous paper that the FCG rate in $0.7 \mathrm{MPa}$ hydrogen becomes maximum at testing frequency of $0.1 \sim 1 \mathrm{~Hz}[11]$. Therefore, we assumed $1 \mathrm{~Hz}$ is preferable to observe typical phenomena of HAFCG also in pure iron.. Moreover, FCG tests were also conducted under the $\Delta K$-constant condition so as to observe the hydrogen effect on the crack propagation morphology at a fixed $\Delta K$ value $\left(\Delta K=17 \mathrm{MPa} \cdot \mathrm{m}^{1 / 2}\right)$.

The fracture surfaces formed in $\triangle P$-constant tests were observed by SEM operated at $15 \mathrm{kV}$. After the $\Delta K$-constant tests, specimens were cut along the mid-thickness section and the surfaces were polished with colloidal $\mathrm{SiO}_{2}$. Crystallographic orientations were analyzed along the fracture path by EBSD, with a beam step size of $1 \mu \mathrm{m}$. Based on the crystal orientation maps, the strain distribution along the cracks was also examined using the grain reference orientation deviation (GROD) method. Here, the average orientation of each grain was used as an orientation reference, with a deviation angle from the reference at every scanned point being mapped for each grain. Additionally, ECCI was conducted with an accelerating voltage of $30 \mathrm{kV}$, in order to obtain an overview of the microstructural evolution along the fracture path. TEM samples perpendicular to the mid-thickness surfaces of the $\Delta K$-constant test specimens were extracted using the JEOL JIB-4500 multi-beam machine. The samples were evaluated using a JEOL 2100F, field-emission-TEM, operated at $200 \mathrm{kV}$ in scanning mode (STEM).

The $\Delta P$-constant tests revealed the existence of two distinctive regions on the $\mathrm{d} a / \mathrm{d} N-\Delta K$ curve in hydrogen, as indicated in Fig. 1: (i) the low $\Delta K$ regime, where the $\mathrm{d} a / \mathrm{d} N$ value was approximately equal 
to that in air (Stage I) and (ii) the high $\Delta K$ regime, with a high acceleration rate (Stage II). Yoshikawa et al. [11] investigated the HAFCG of a low-carbon steel in hydrogen gas at various pressures, reporting that the material exhibited an upper limit of FCG acceleration (an acceleration rate approximately 30 times greater than the FCG rate in air), regardless of testing frequency, viz., the material exhibited no tendency of delayed cracking and the strain-controlled fracture was dominant even in hydrogen atmosphere. FCG rates in the low-carbon steel, as obtained in the same environments [11], were also plotted in Fig. 1. The FCG curves are consistent with each other, indicating that the crack propagated accordingly via the identical mechanism in the two materials, namely strain-controlled fracture.

Figure 2 presents the fracture surfaces observed via SEM. Whereas ductile striations were formed throughout the entire $\Delta K$ range in air, the fracture morphology in hydrogen changed dramatically. Figure 2 (b) reveals the fracture surface in Stage I, as formed in hydrogen. Even though there was no acceleration of FCG at this stage, surprisingly, almost half of the fracture surface was covered by intergranular (IG) facets with a brittle appearance while none of IG features were visible in case of air. The IG fracture in HAFCG was also reported in previous researches [4, 25, 26]. However, even though the IG facets seemed brittle macroscopically, numerous slip traces were found at higher magnifications (region A in Fig. 2 (b)), implying the mediation of plasticity during the fracture process. Martin et al. [21] reported the formation of similar slip traces on the IG fracture surface of hydrogen-charged pure $\mathrm{Ni}$, and concluded that dislocation movements play an important role in the grain boundary (GB) separation. Specifically, the hydrogen distributed inside the grain was transported by moving dislocations, then deposited into the GBs, in conjunction with the piling-up of dislocations, resulting in the local satisfaction of stress concentration and hydrogen agglomeration for GB decohesion [27]. Similar explanations may be applicable in the present case, although the direct correlation between the IG fracture and the macroscopic FCG rate remains unclear and the in depth analysis of stage I FCG is beyond the scope of this article.

In association with the onset of FCG acceleration, an abrupt transition of fracture morphology was observed in hydrogen (Fig. 2 (c)). In contrast to the IG fracture in Stage I, the fracture surface in Stage II was covered by faceted, transgranular planes (quasi-cleavage $=\mathrm{QC}$ ), frequently observed on the hydrogen-affected fracture surfaces of low- to medium-strength steels $[1,8,11]$. Moreover, a magnified image of this QC (region B in Fig. 2 (c)) revealed the existence of brittle-like striations which ran perpendicular to the macroscopic FCG direction. It was confirmed that the average striation spacing was roughly consistent with the macroscopic FCG rate at the corresponding $\Delta K$ value. Chen and Gerberich [28] reported similar striations on $\{100\}$ cleavage planes of Fe-3\%Si single crystals during sustained-load experiments in hydrogen, as the evidence of intermittent, time-dependent, crack propagation. However, consistency of striation-spacing and the FCG rate in the present study imply that the striations were formed on a cycle-by-cycle basis, and their formation mechanism was different from that reported by Chen and Gerberich. The formation mechanism of such brittle-like striations in hydrogen was studied by Matsuoka et al. [10, 29]. They tested several carbon steels and Cr-Mo steels in hydrogen, reporting that 
the striations were the consequence of hydrogen-induced reduction of the plasticity expansion in crack-tip region(HISCG). Details about that model are documented in other literature [10, 29].

In order to validate the previous assumptions, i.e., the reduction of plastic flow due to hydrogen, $\Delta K$-constant tests were performed in a Stage II regime. The results are plotted together with the results of the $\Delta P$-constant tests in Fig. 1. Significant FCG acceleration was also observed in the $\Delta K$-constant tests, nearly equivalent to that established in the $\Delta P$-constant test. The results of EBSD and ECCI analyses of the mid-thickness sections of specimens after the $\Delta K$-constant-test are exhibited in Fig. 3. The ECC images in Figs. 3 (e) and (f) correspond to the area surrounded by white dotted lines in Figs. 3 (a) and (b), respectively. In air, grain orientations near the fracture path were heavily distorted and clear contrast changes were observed in a corresponding ECC image (Fig. 3 (a) (e)), implying the formation of sub-grains or dislocation cell structures. However, in hydrogen, very little grain distortion was observed and only a slight contrast change was visible in the ECC image (Fig. 3 (b) (f)). The GROD maps in Figs. 3 (c) and (d) reflects these differences more intuitively. When comparing Figs. 3 (c) and (d), the extent of plastic deformation along the fracture path was definitely seen to be weaker in the specimen tested in hydrogen. These novel results directly support the hypothesis that hydrogen-enhanced reduction of crack-tip plasticity expansion is the main contributing factor in strain-controlled HAFCG, as proposed in our previous studies [10, 11, 29].

Furthermore, as exhibited in the GROD maps, plastic deformation was uniformly distributed along the crack path in air (Fig. 3 (c)), whereas the deformation lacked uniformity in hydrogen (Fig. 3 (d)). Thus, only one TEM sample was extracted from a specimen tested in air and two TEM samples from specimens tested in hydrogen. The one from area "A" was considerably plastically-deformed while the other from area "B" was relatively free of plastic deformation. Figure 4 presents dark-field, STEM micrographs of these samples. The exact locations of the samples are marked by yellow rectangles in Figs. 3 (e) and (f). The specimen tested in air (Fig 4. (a)) succumbed to a dislocation in cell structure at a depth of about 3 $\mu \mathrm{m}$ beneath the fracture surface. Further away, a transition into a dislocation wall/vein structure was observed. A sample extracted from area " $\mathrm{A}$ " showed similar dislocation structures as the specimen tested in air. However, the cell structure ranged from about $2 \mu \mathrm{m}$ from the fracture surface. In contrast, the sample extracted from area "B" displayed only individual dislocations and dislocation tangles scattered around. No dislocation cell, wall or vein structure was detected.

The evolution of dislocation structure formation during fatigue is well summarized by Polák [30], who states that the type and proportion of dislocation structures depend on applied plastic strain amplitude. In general, cyclic loading at low plastic strain amplitudes results in a planar dislocation arrangement; when loading at medium amplitudes, the random arrangement transforms into vein and wall structures, whereas high-amplitude loading leads to the formation of dislocation cells structures.

Figures 4 (a) and (b) demonstrate that plastic strain is sufficiently high to form cells in both atmospheres. However, in hydrogen, the cell structure is more localized when compared to specimens 
tested in air. These results are in good agreement with Martin et al. [20] who showed that hydrogen localizes the dislocation cell structure closer to the fracture surface, also reducing the cell size in Type 304 stainless steel. Such enhancement of the deformation processes was attributed to the hydrogen acceleration of the plastic processes via shielding dislocation strain fields [15], thereby increasing their mobility [14].

In contrast to the cell and vein/wall formation shown in Fig. 4 (b), Fig. 4 (c) reveals no development of dislocation structures, but rather a random distribution of individual dislocations and dislocation tangles. According to the HELP mechanism, hydrogen is transported by mobile dislocations $[31,32]$ and the formation of dislocation tangles may result in the enhancement of local hydrogen concentrations, potentially enough to trigger lattice failure at lower plastic strain amplitudes and to suppress the further development of dislocation structures. The diffraction pattern in the inset indicates that the fracture surface corresponds to the $\{100\}$ type cleavage plane. Since the $\{100\}$ plane is already at its weakest in BCC crystal, it becomes a preferable fracture plane at some critical hydrogen concentrations. However, the image indicates that it is not a perfectly flat plane, but instead demonstrates some unevenness. Martin et al. [33] studied hydrogen-induced, flat fracture surfaces in pipeline steels. They observed very similar dislocation structures beneath the surface and used the HELP model to explain the development of dislocation structures which result in increased hydrogen concentrations, later increasing the propensity for cracking. In addition, atomic force microscopy confirmed the existence of an undulated surface and not one as perfectly flat as it had appeared under SEM [33]. This conclusion is in good agreement with our observations. Considering those earlier results and the dislocation structures observed in this study, it can be inferred that both of the formation of localized cell structure due to HELP (Fig. 4 (b)) and HELP-mediated cleavage-like fracture (Fig. 4 (c)) are responsible for the FCG acceleration in pure iron in the Stage II regime.

FCG tests of a pure Fe were performed in a $0.7 \mathrm{MPa}$ hydrogen gas in order to elucidate the influence of hydrogen on the plastic deformation process near the fatigue crack-tip. Beyond a specific $\Delta K$ value, the FCG rate was significantly accelerated in the presence of hydrogen, with the corresponding fracture morphology transiting to QC. EBSD, ECCI and TEM techniques were applied to the mid-thickness fracture path of the CT specimens. In consequence, EBSD and ECCI successfully demonstrated the hydrogen-enhanced reduction of plasticity expansion on a microscale, supporting the model which had been developed during our previous investigations. TEM observation confirmed a difference in the dislocation structures located close to the fracture surface. Sample fatigued in air bore a dislocation cell structure, whereas the samples fatigued in hydrogen possessed a localized cell structure in the highly-deformed area and a planar dislocation structure in the least deformed area. The preceding results illustrate that the reduction of crack-tip plasticity expansion contributes to the FCG acceleration in a gaseous hydrogen environment. 
This work was supported by JSPS KAKENHI Grant Number 16H04238 and 16J02960. The Research Council of Norway is acknowledged for its support through the Norwegian Center for Transmission Electron Microscopy, NORTEM (197405/F50). This study also forms part of the "HIPP" project from the PETROMAKS2 program, funded by the Research Council of Norway [Grant Number: 102006899].

\section{References}

[1] H.J. Cialone, J.H. Holbrook, Metallurgical Transactions A 16 (1985) 115-122.

[2] A.J. Slifka, E.S. Drexler, N.E. Nanninga, Y.S. Levy, J.D. McColskey, R.L. Amaro, A.E. Stevenson, Corrosion Science 78 (2014) 313-321.

[3] Z. Sun, C. Moriconi, G. Benoit, D. Halm, G. Hénaff, Metallurgical and Materials Transactions A 44 (2013) 1320-1330.

[4] B.P. Somerday, P. Sofronis, K.A. Nibur, C. San March, R. Kirchheim, Acta Materialia 61 (2013) 6153-6170.

[5] C.J. McMahon Jr., Engineering Fracture Mechanics 68 (2001) 773-788.

[6] R.P. Wei and J.D. Landes, Materials Research \& Standards 9 (1969) 25-46.

[7] I.M. Austen and P. Mclntyre, Metal Science 13 (1979) 420-428.

[8] T.J. Marrow, P.J. Cotterill, J.E. King, Acta Metall. Mater. 40 (1992) 2059-2068.

[9] Y. Murakami, T. Kanezaki, Y. Mine, S. Matsuoka, Metallurgical and Materials Transactions A 39 (2008) 1327-1339.

[10] S. Matsuoka, H. Tanaka, N. Homma, Y. Murakami, International Journal of Fracture 168 (2011) 101-112.

[11] M. Yoshikawa, T. Matsuo, N. Tsutsumi, H. Matsunaga, S. Matsuoka, Transactions of the Japan Society of Mechanical Engineers 80-817 (2014).

[12] J. Yamabe, M. Yoshikawa, H. Matsunaga, S. Matsuoka, Procedia Structural Integrity 2 (2016) 525-532.

[13] S. Matsuoka, J. Yamabe, H. Matsunaga, Engineering Fracture Mechanics 153 (2016) 103-127.

[14] T. Tabata, H.K. Birnbaum, Scripta Metallurgica 17 (1983) 947-950.

[15] I.M. Robertson, Engineering Fracture Mechanics 68 (2001) 671-692.

[16] H. Nishikawa, Y. Oda, H. Noguchi, Journal of Solid Mechanics and Materials Engineering 5 (2011) 370-385.

[17] C.D. Beachem, Metallurgical Transactions 3 (1972) 437-451.

[18] Y. Takahashi, M. Tanaka, K. Higashida, H. Noguchi, Scripta Materialia 61 (2009) 145-148.

[19] S. Wang, M.L. Martin, P. Sofronis, S. Ohnuki, N. Hashimoto, I.M. Robertson, Acta Materialia 69 (2014) 275-282.

[20] M. Martin, P. Sofronis, I. Robertson, T. Awane, Y. Murakami, International Journal of Fatigue 57 (2013) 28-36. 
[21] M.L. Martin, B.P. Somerday, R.O. Ritchie, P. Sofronis, I.M. Robertson, Acta Materialia 60 (2012) 2739-2745.

[22] Y. Takahashi, J. Sakamoto, M. Tanaka, K. Higashida, H. Noguchi, Scripta Materialia 64 (2011) 721-724.

[23] Y. Takahashi, M. Tanaka, K. Higashida, K. Yamaguchi, H. Noguchi, Acta Materialia 58 (2010) 1972-1981.

[24] ASTM, E647-08-1. Standard test method for measurement of fatigue crack growth rates. West Conshohocken: ASTM International; 2010.

[25] G. Bilotta, M. Arzaghi, G. Hénaff, G. Benoit, D. Haim, in: Proceedings of the ASME 2016 Pressure Vessels and Piping Conference (PVP2016), No. 63338, 2016.

[26] M. Koyama, Y. Onishi, H. Noguchi, International Journal of Fracture 206 (2017) 123-130.

[27] I.M. Robertson, P. Sofronis, A. Nagao, M.L. Martin, S. Wang, D.W. Gross, K.E. Nygren, Metallurgical and Materials Transactions B 46 (2014) 1085-1103.

[28] X. Chen, W.W. Gerberich, Metallurgical Transactions A 22 (1991) 59-70.

[29] S. Matsuoka, N. Tsutsumi, Y. Murakami, Transactions of the Japan Society of Mechanical Engineers 74 (2008) 1528-1537.

[30] J. Polák, 4.20 - Fatigue of Steels, in: Comprehensive Structural Integrity, I. Milne, R.O. Ritchie, B. Karihaloo (Eds.), Pergamon, Oxford, 2007, pp. 504-537, ISBN 9780080437491.

[31] J.K. Tien, A.W. Thompson, I.M. Bernstein, R.J. Richards, Metallurgical Transactions A 7 (1976) 821-829.

[32] M. Dadfrania, M.L. Martin, A. Nagao, P. Sofronis, I.M. Robertson, Journal of the Mechanics and Physics of Solids 78 (2015) 511-525.

[33] M.L. Martin, I.M. Robertson, P. Sofronis, Acta Materialia 59 (2011) 3680-3687. 